\title{
Selection of optimal combinations of loci by the Optimal Combination Finder computer program from a group of variable number tandem repeat loci for use in Staphylococcus aureus food poisoning case investigations
}

\author{
Xian Wang, ${ }^{1} \dagger$ Bixing Huang, ${ }^{2}+$ Barry Blair, $^{2}$ Sofroni Eglezos ${ }^{3}$ \\ and John Bates ${ }^{2}$ \\ ${ }^{1}$ Shijiazhuang Center for Disease Control and Prevention, 3 Likang Street, Shijiazhuang, \\ Hebei Province, PR China \\ ${ }^{2}$ Queensland Public Health Microbiology Laboratory, Forensic and Scientific Services, \\ 39 Kessels Road, Coopers Plains, QLD 4108, Australia \\ ${ }^{3}$ EML Consulting Services OLD, 1/148 Tennyson Memorial Avenue, Tennyson, OLD 4105, \\ Australia
}

Correspondence

Xian Wang

wangxia718@163.com

Bixing Huang

Ben_Huang@health.qld.gov.au

Received 1 November 2011

Accepted 14 January 2012

\begin{abstract}
Staphylococcus aureus is a pathogen that can be the cause of nosocomial infections, communityacquired diseases and food-borne disease outbreaks. A number of variable number tandem repeat loci have been reported by various groups for use in epidemiological studies and outbreak investigations. The aim of this study was to systematically evaluate a total of 18 commonly used loci with the same $S$. aureus population so that the properties of each locus could be compared and used for typing, and to develop a computer program enabling calculation of the Simpson index (SI) of all possible loci combinations so that an optimal combination of loci could be identified for multilocus variable-number tandem-repeat analysis (MLVA) typing. A collection of 160 S. aureus isolates from patients with sporadic food-borne illnesses, and pet animals, such as canine, feline and equine pets, with skin infections were used to assess the MLVA loci. The newly developed Optimal Combination Finder (OCF) computer program was used for the analysis and it was found that the minimal number of loci combinations that produced the same $\mathrm{SI}(0.999528)$ as that of the 18 loci combined was eight; SIRU05, SIRU07, SIRU13, SIRU15, SIRU21, fnbB, sdrD and $c / f B$. This suggested that the optimal combination of eight loci could be used for the routine investigation and surveillance of future $S$. aureus outbreaks instead of using all 18 loci. In addition, the OCF software could be a useful tool for the development of typing schemes for other organisms.
\end{abstract}

\section{INTRODUCTION}

Staphylococcus aureus is found on the skin, mucous membranes and hair of warm-blooded animals. Between 30 and $50 \%$ of the human population are carriers (Bannerman \& Peacock, 2007). S. aureus can grow in a wide range of temperatures $\left(7-48.5{ }^{\circ} \mathrm{C}\right.$, optimum 30 $37^{\circ} \mathrm{C}$; Schmitt et al., 1990), $\mathrm{pH}$ levels ( $\mathrm{pH}$ 4.2-9.3, optimum $\mathrm{pH} 7-7.5$ ) and $\mathrm{NaCl}$ concentrations (up to $15 \%$, w/v, NaCl; Le Loir et al., 2003). These properties enable $S$. aureus to grow in a wide variety of foods, making

†These authors contributed equally to this work.

Abbreviations: MLVA, multiple locus variable number tandem repeat analysis; OCF, Optimal Combination Finder; SI, Simpson index; SIRU, staphylococcal interspersed repeat unit. it one of the leading pathogens with regard to food-borne diseases (Asao et al., 2003; Cowell et al., 2002; Fullerton \& OzFoodNet Working Group, 2009). In addition, S. aureus has a combination of virulence factors including toxin production, invasiveness and antibiotic resistance, making it a significant cause of nosocomial and communityacquired infection (Coombs et al., 2009; Hardy et al., 2004; Vindel et al., 2009).

A number of DNA-based typing methods have been developed for the investigation and epidemiological surveillance of $S$. aureus outbreaks and strain dissemination. Typing schemes using multilocus variable-number tandem-repeat analysis (MLVA) of S. aureus have been reported by various groups (Conceição et al., 2009; Francois et al., 2005; Gilbert et al., 2006; Hardy et al., 
2006; Melles et al., 2009; Pourcel et al., 2009; Sabat et al., 2003; Schouls et al., 2009). Comparisons of MLVA studies with other $S$. aureus typing methods have found that MLVA has advantages over pulsed-field gel electrophoresis (PFGE), spa typing and amplified fragment length polymorphism (AFLP) methods in reproducibility, discriminatory power and cost (Malachowa et al., 2005; Melles et al., 2009; Tenover et al., 2007; Vindel et al., 2009). MLVA is regarded as a well-recognized typing method (Lindstedt, 2005; van Belkum, 2007). MLVA provides genotypes expressed in strings of numbers corresponding to the number of repeats at each locus; therefore, this typing method produces results that are highly portable and easy to incorporate into an established database (Grissa et al., 2008).

Reports of $S$. aureus MLVA in the literature are chiefly based on the following variable tandem repeats: staphylococcal interspersed repeat units (SIRUs), including SIRU01, -05, -07, -13, -15,-16, and -21 (Hardy et al., 2006); intergenic and S. aureus repeats (STAR), such as 311 (Pourcel et al., 2009); and tandem repeats within genes such as clfA, clfB, sdrCDE, spa and sspA (Sabat et al., 2003). In the present study, a total of 18 MLVA loci were selected and assessed using a collection of $S$. aureus strains from our laboratory. These loci are frequently used and have been proven to be polymorphic in various reports. Authors validated these loci based on their own limited populations. Therefore, the present study aimed to compare these existing typing loci among strains of the same $S$. aureus population to identify optimum loci for use in future foodborne outbreak investigations.

As a large number of $S$. aureus MLVA loci have been described to date, the choice of the most appropriate combination of loci for typing is not obvious. The number of loci for typing must be reduced to a more practical number for routine use. To select an optimal combination of loci, ideally, the discriminatory ability of every possible combination of these loci should be determined. However, this approach is not feasible by manual calculation, as even for a five-locus combination, selected from 18 candidate loci, there are 8568 possible combinations. Therefore, a computer program was developed to quantify the ability of each possible combination of loci to discriminate between strains, making it possible to efficiently select the optimal combination of candidate loci. The aim of this study was to compare 18 commonly used MLVA markers in the same $S$. aureus population for assessment of the loci for future analysis, and to develop a computer program for the identification of an optimal combination of loci for use in future $S$. aureus epidemiological investigations and outbreak studies.

\section{METHODS}

S. aureus samples. A total of 160 epidemiologically unrelated isolates were used in this study. Of the isolates, 38 were unassociated isolates from documented sporadic food poisoning cases in
Queensland from 2000 to 2008 , which were stored at $-80{ }^{\circ} \mathrm{C}$, and the remaining 122 were animal isolates collected from February to October 2009 and were obtained from Idexx Laboratories, Coorparoo, Queensland, a veterinary microbiology testing laboratory. The animal samples were from abscesses, skin lesions/wounds, nose discharge and milk of pets and zoo animals. The pet animal isolates comprised 63 canine, 21 feline, 19 equine, five bovine, three rabbit, one chicken and three goat isolates. Zoo animal isolates comprised four possum, one koala, one monkey and one wallaby isolates. Since pet and zoo animals have close contact with humans, isolates from pets and zoo animals were included to extend the S. aureus source representation for the MLVA marker assessment. Two reference strains of S. aureus, ATCC 6538 and NCTC 4163, were passaged using blood plates to assess the stability of the loci. Isolates at passages 1,5 , 10,15 and 20 were tested for variability in the 18 loci.

To test the concordance of the MLVA markers in outbreak investigations, another 18 isolates from two known outbreaks were analysed (see Table 4). Detection of 19 enterotoxin genes was carried out for these outbreak isolates according to the method described by Hwang et al. (2007).

Choice of markers. The MLVA loci used by Hardy et al. (2006) and Sabat et al. (2003) were selected as they have been widely used in other studies. An additional five loci (coa, SAV1078, 311, fnbA and $f n b B)$, used in a number of previous studies, were evaluated against the 13 sequenced genomes of Staphylococcus aureus that were publicly available in GenBank (http://www.ncbi.nlm.nih.gov/genbank/) at the time of this project. These loci were variable among the sequenced genomes of the 13 strains: COL, USA300F, MSSA476, MRSA252, RF122bov, Mu50, MW2, N315, NCTC8325, SubJH1, SubJH9, SubMu3 and Newman.

Primer design. Due to their high sequence similarity, the three genes $s d r C, s d r D$ and $s d r E$ are amplified in one reaction using the primers designed by Sabat et al. (2003); therefore, it was impossible to assign the amplified bands to $s d r C$, $s d r D$ or $s d r E$ in an Excel file for analysis. Because of this, efforts were made to design specific primers for amplification of each of the $s d r C, s d r D$ and $s d r E$ genes separately. To differentiate the amplification products further, forward primers for the $s d r C, s d r D$ and $s d r E$ genes were labelled with different coloured dyes, FAM, HEX and NED, respectively. The individually amplified products could be distinguished by their colours using an ABI Genetic Analyzer 3130 . The products were sequenced to check the amplification specificity. This was also the case for the $f n b A$ and $f n b B$ genes. As the primers for the SIRU21 locus and spa genes targeted the same variable region, only the results of SIRU21 were presented here (Pourcel et al., 2009; Sabat et al., 2003). The primers used in this study are listed in Table 1.

DNA extraction. Genomic DNA was extracted using a MasterPure DNA extraction kit (Epicentre) following the manufacturer's instructions with a modification of the cell lysis step, which was performed with $50 \mathrm{U} \mathrm{ml}^{-1}$ lysostaphin (Sigma). The DNA was measured using a spectrophotometer (Gene Quant II, Pharmacia Biotec) at $A_{260}$ and the DNA was made up to a concentration of $50 \mathrm{ng} \mu \mathrm{l}^{-1}$.

PCR conditions. To save time and reagents, multiplex PCR was applied wherever possible. The combinations were as follows: set 1: SIRU01, SIRU07 and SIRU16; set 2: SIRU05 and SIRU21; set 3: SIRU15 and $c l f B$; set 4: spa and sspA; set 5: $f n b B$ and $s d r E$; set 6: SIRU13 and SAV1078; set 7: 311 and $f n b A$. Single PCRs were performed for $s d r C, s d r D$, coa, and $c l f A$ as multiplex PCR did not yield good results. A confirmation single PCR was carried out for the samples whose locus/loci were negative in the multiplex PCRs. Each PCR mixture contained $2 \mu \mathrm{l}$ prepared template DNA, $0.4 \mu \mathrm{M}$ each primer, $2.5 \mu \mathrm{l}$ 
Table 1. Loci and primer sequences used in this study

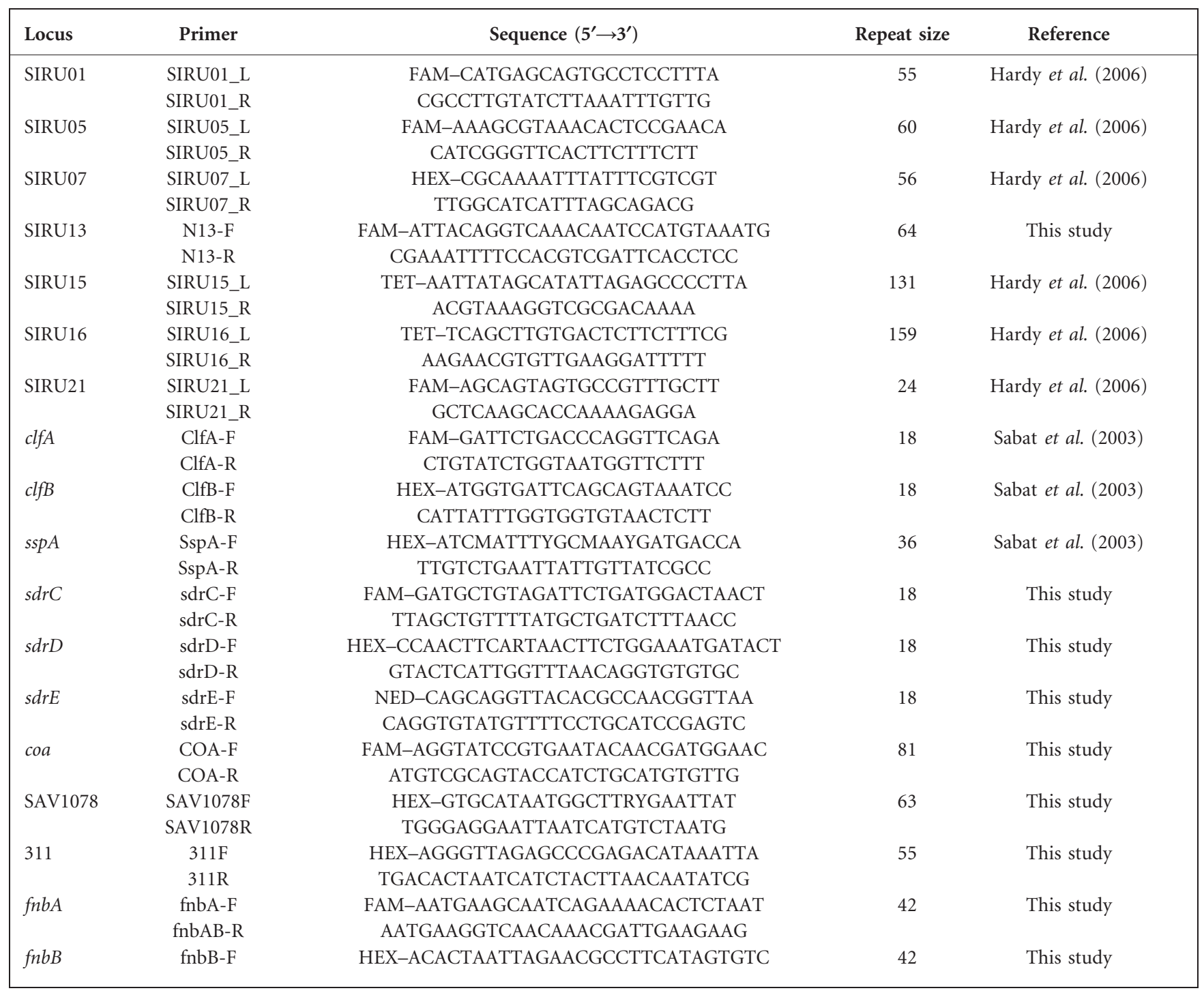

$10 \times$ buffer II (Applied Biosystems), $2.5 \mathrm{mM} \mathrm{MgCl}_{2}, 200 \mu \mathrm{M}$ each dATP, dCTP, dGTP and dTTP (GE Healthcare), $1.25 \mu \mathrm{l}$ DMSO (Sigma) and $0.5 \mathrm{U}$ AmpliTaq Gold DNA polymerase (Applied Biosystems). PCR was performed in a GeneAmp 9700 PCR machine (Applied Biosystems) using the following cycling conditions: $95{ }^{\circ} \mathrm{C}$ for $10 \mathrm{~min}$, followed by 30 cycles of $94{ }^{\circ} \mathrm{C}$ for $30 \mathrm{~s}, 55^{\circ} \mathrm{C}$ for $30 \mathrm{~s}$ and $72{ }^{\circ} \mathrm{C}$ for $1 \mathrm{~min}$, with a final extension step at $72{ }^{\circ} \mathrm{C}$ for $7 \mathrm{~min}$. After PCR amplification, the fragments were separated by capillary electrophoresis on an ABI 3130 Genetic Analyzer (Applied Biosystems). The sizes were determined by using a $50-1000$ bp internal standard marker (MapMarker 1000, BioVentures) and Peak Scanner software version 1.0 (Applied Biosystems).

After analysing the results of the 18 loci, the optimal combination of loci was identified to be an eight-locus combination of SIRU05, SIRU21, SIRU13, SIRU15, clfB, fnbB, SIRU07 and $s d r D$. The eight loci were optimized and rearranged in the following multiplex PCRs: set 1: SIRU05, SIRU21 and SIRU13; set 2: SIRU15, clfB and $f n b B$; and set 3: SIRU07 and $s d r D$. A string of eight numbers starting in the same order as the eight loci was used to express the profile of an isolate.

The allele coding system. To calculate the number of repeats in an allele, the amplicon size in base pairs was recorded. The two flanking regions were then subtracted from the amplicon length. The resulting figure divided by the repeat unit length was the number of repeats. For partial repeats, if the partial repeat was less than half the size of the repeat, they were included in the flanking regions as recommended by Hardy et al. (2004), and when the partial repeat was larger than half of the repeat it was rounded up to a full repeat. In addition, it has been observed that some strains have flanking regions but do not contain repeat units (Hardy et al., 2004); therefore, an amplicon was still produced. In some strains, the flanking regions and the repeat unit were not present in the genomes; therefore, no PCR product was observed (Ikawaty et al., 2008). To accommodate and differentiate between these different scenarios, a numerical coding system was used. The result was expressed as 0 if there was no amplicon (i.e. the locus was absent); 1 if the size of the amplicon corresponded with that of the flanking region (i.e. the locus is present but no repeat sequence was present); 2 if the amplicon length corresponded with the sum of the flanking region and one repeat, and so on. This numerical coding system was used for all 18 loci studied. The string of 18 numbers gave an allelic profile for each $S$. aureus isolate.

The development of the Optimal Combination Finder (OCF) program. The discriminatory power of a typing method is in its 


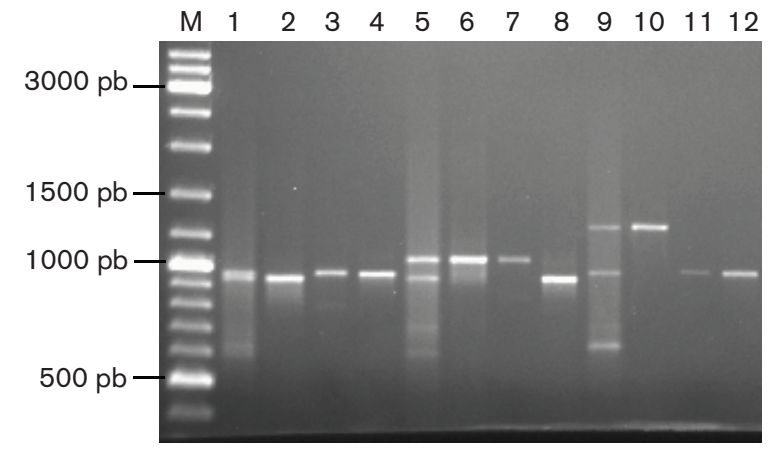

Fig. 1. Comparison of the PCR products amplified by the previously designed $s d r C D E$ primers (lanes 1, 5, 9) with the newly designed primers for the individual sdrC (lanes 2, 6, 10), $s d r D$ (lanes 3, 7, 11) and sdrE (lanes 4, 8, 12) genes. Lanes: M, GeneRuler (Fermentas); 1-4, sample 09M1304; 5-8 sample 09M1305; 9-12, sample 09M1306. In sample 09M1304, the amplified PCR products of $s d r C$, sdrD, and $s d r E$ (lanes 2-4) were similar sizes and the amplified PCR products of $s d r C D E$ (lane 1) could not be separated well; therefore, only a single band was observed. In sample 09M1305, sdrC and sdrD (lanes 6 and 7) were a similar size, whereas $s d r E$ produces a band of different size. As a result, only two bands were observed for sdrCDE (lane 5). In sample 1306, sdrD and sdrE (lanes 11 and 12) were a similar size, whereas $s d r C$ was a different size. Therefore, only two bands were observed and the smallest of these ( $600 \mathrm{bp})$ might have been a non-specific band. The newly designed primers for $s d r C, s d r D$ and $s d r E$ have improved the PCR specificity and made the use of the variability of these loci possible.

ability to distinguish between unrelated strains and is expressed as the Simpson index (SI) (Hunter \& Gaston, 1988). This is dependent on the types defined by the test method and the relative frequencies of these types. In the present study, the discriminatory power of the MLVA method was calculated using a previously described formula (Hunter \& Gaston, 1988). To facilitate the choice of an optimal combination of loci, the SI was calculated for every possible combination of loci using the Optimal Combination Finder (OCF) computer program, which was developed for this study. The program was written using Visual Basic (VBA) 2007 to derive every possible combination and was used in conjunction with Microsoft Excel using a series of cascading loops to calculate SI for every possible combination of loci. The SI results calculated by the OCF program were ranked in a list of loci combinations in order of highest to lowest. By systematically stepping through sets of 2-18 loci, the optimal combination of loci could be defined as the point at which the SI did not increase when more loci were selected into the combination. The optimal combination of loci consisted of the minimal number of loci producing the maximal SI so that minimal work load is required to obtain optimal results.

\section{RESULTS}

\section{The VNTR loci}

Three sets of loci with high sequence similarity, close locality and similar functionality were included in this study. These were $s d r C, s d r D$ and $s d r E$; $c l f A$ and $c l f B$; and $f n b A$ and $f n b B$. Attempts were made to design specific primers to amplify these genes individually. Fig. 1 shows that the original $s d r C D E$ primers produced multiple bands and the newly designed primers could amplify the $s d r C$, $s d r D$ and $s d r E$ genes individually. In addition, the forward primers for amplification of the $s d r C, s d r D$ and $s d r E$ genes were labelled with FAM, HEX and NED, respectively, making it easier to differentiate between them in the ABI 3130 Genetic Analyzer. The amplified PCR products could, therefore, be assigned to their corresponding genes, making the information from these polymorphic loci useful in the analysis (Table 2).

For the 18 loci, the number of alleles observed was between three alleles at SIRU16 and 28 alleles at clfB. The discriminatory power, expressed in SI, for each individual locus is listed in Table 2. The highest SI value was 0.935 for $c l f B$, and the lowest SI was 0.506 for SIRU01. The stability of the VNTR loci was tested by analysing the allele sizes on passages 1, 5, 10, 15 and 20 of S. aureus strains ATCC 6538 and NCTC 4163. No variations in allele size were observed, suggesting that the loci were stable within the passages tested (data not shown).

\section{The choice of the MLVA panel}

The discriminatory power of MLVA increases with the number of polymorphic loci included in the typing panel. Normally, loci are chosen based on their SI value and the individual loci with the highest SI are combined to form the MLVA testing panel. However, the best loci may not complement each other and may provide redundant information. For example, to make a three-locus combination out of the 18 loci studied, the conventional method would be to select the three individual loci with the highest SI ( $c l f B, c l f A$ and $s d r E$ ) to form the locus combination arbitrarily (Table 2), giving an SI of 0.99387 . It is, however, hard to know if this is the optimal combination as the SIs of the remaining possible combinations from the 18 loci are not calculated and compared. From the 18 loci studied, there were a possible 816 kinds of threelocus combination. It is not logistical to manually calculate every combination. A computer program termed 'Optimal Combination Finder' (OCF) was developed to provide a solution to this problem. The program calculated the SI of 816 kinds of three-locus combination and ranked the SI values in order from the highest to the lowest. In this case, the optimal combination of three loci was clfB, clfA and SIRU21 with an SI of 0.99599 (Table 3), which was higher than that of our empirically selected combination of $\operatorname{clf} B$, clfA and sdrE (SI 0.99387).

When a request was made to find the optimal combination of four loci, this program made a new calculation independently from the previous three-locus calculations. It calculated every possible combination of four loci from the 18 loci used in this study. The optimal combination of four loci was clfB, SIRU05, SIRU13 and SIRU21, as determined by the OFC program. A normal human 
Table 2. Alleles and SI of the individual loci

\begin{tabular}{|c|c|c|c|c|}
\hline Loci & Range of PCR products (bp) & No. of alleles observed & Null allele percentage & SI \\
\hline $\operatorname{clfB}$ & $552-1119$ & 28 & $0.0 \%$ & 0.935 \\
\hline clfA & $682-1158$ & 23 & $1.2 \%$ & 0.896 \\
\hline$s d r D$ & $789-1126$ & 20 & $23.1 \%$ & 0.883 \\
\hline SIRU21 & $119-490$ & 13 & $0.0 \%$ & 0.858 \\
\hline SIRU13 & $208-876$ & 11 & $0.6 \%$ & 0.856 \\
\hline SIRU05 & 203-1045 & 13 & $28.7 \%$ & 0.839 \\
\hline SspA & $67-216$ & 7 & $0.0 \%$ & 0.786 \\
\hline$f n b A$ & $514-936$ & 9 & $20.6 \%$ & 0.783 \\
\hline 311 & $252-552$ & 8 & $0.6 \%$ & 0.774 \\
\hline$f n b B$ & $469-808$ & 8 & $0.6 \%$ & 0.742 \\
\hline SIRU07 & $246-417$ & 5 & $13.1 \%$ & 0.716 \\
\hline SIRU01 & $237-407$ & 4 & $3.1 \%$ & 0.506 \\
\hline
\end{tabular}

empirical choice would have been based on the assumption that the loci from the optimal three-locus combination would still perform well in a four-locus combination; therefore, the previous optimal three-locus combination $c l f B, c l f A$ and SIRU21 would be kept and an extra locus would be added to form a four-locus combination. However, only two loci from the original three-locus combination, $c l f B$ and SIRU21, were included in the optimal four-locus combination as determined by the OFC program (Table 3 ). This suggests that the program overcame this human assumption and identified the optimal combination based on calculations of every possible combination.

Fig. 2 shows the SI values of the optimal locus combinations and the poorest locus combinations of 2-18 loci as identified by the OCF program. SI values based on a human empirical choice of loci for these combinations would most likely fall between these two curves and would probably require more empirical testing to achieve the same SI values as those of the optimal locus combinations.

A series of tests were carried out on combinations of 2-18 loci and it was found that a maximum SI of 0.999528 was observed with eight optimally selected loci: SIRU05, SIRU07, SIRU13, SIRU15, SIRU21, fnbB (or $f n b A$ ), $s d r D$ and $c l f B$. No increase of SI value was observed by the addition of extra loci to this set (Table 3 ). This means that the eight selected loci represent the minimal number of loci required to produce the maximum SI. In the optimal eightlocus combination, $f n b B$ was selected over $f n b A$ as $f n b B$ had a lower null allele rate than that of $f n b A$ (Table 2).

The discriminatory power of the loci reported by Hardy et al. (2004) and Sabat et al. (2003) for the samples in this study was assessed. Using the seven loci of Hardy et al. (2004), 140 genotypes with an SI of 0.9980 were observed, and using the five loci of Sabat et al. (2003), 140 genotypes with an SI of 0.9971 were observed. These data suggest that the optimal eight-locus combination identified in the present study could produce more genotypes (154 genotypes observed) than that of either of the sets of loci reported by Hardy et al. (2004) and Sabat et al. (2003).

\section{The case investigations}

Stored isolates from two epidemiological investigations of S. aureus food poisoning outbreaks in Queensland were tested using the eight optimal MLVA loci (Table 4). The first outbreak investigation took place in 2000. The profiles of two isolates suspected to be from contaminated chicken salad were the same, except that for Chicken salad P252B00 sample A the $f n b B$ locus was allele 5 and for Chicken salad P252B00 sample B the $f n b B$ locus was allele 4. Interestingly, the isolates from patients, 00M1063-1, 00M1039-1 and 00M1039-2, had the same MLVA pattern as that of the food isolate Chicken salad P252B00 sample A. The profile of the other isolate from patient 1 (00M1063-2) was the same as that of isolate Chicken salad P252B00 sample B. In addition, all isolates in this case investigation had the same four enterotoxin genes, sea, sek, seh and seq, out of the 19 enterotoxin genes tested for (Hwang et al., 2007). The MLVA profiles of isolates from the suspected food matched the profiles of isolates from patients, suggesting that the food contained different populations of $S$. aureus and subsequently the two different genotypes of the isolates were reflected in patient isolates.

The second outbreak investigation took place in 2008. The MLVA profiles from the isolates of suspected food and the nasal swab of a food catering staff member were the same, except that the MLVA profile of the isolates cream P891/08 and roast chicken P888/08 was allele 34 at the $s d r D$ locus 
Table 3. The highest five loci or loci combinations ranked after the computer program calculation

\begin{tabular}{|c|c|c|c|c|c|c|c|c|c|c|c|}
\hline \multirow{2}{*}{$\begin{array}{l}\text { No. loci } \\
\text { (no. possible } \\
\text { combinations) }\end{array}$} & \multicolumn{9}{|c|}{ The five loci combinations produced the best SI values in descending order } & \multirow{2}{*}{$\begin{array}{c}\text { Genotypes } \\
28\end{array}$} & \multirow{2}{*}{$\begin{array}{c}\text { SI } \\
0.93498\end{array}$} \\
\hline & $c l f B$ & & & & & & & & & & \\
\hline & clfA & & & & & & & & & 23 & 0.89638 \\
\hline & $s d r E$ & & & & & & & & & 17 & 0.88939 \\
\hline & $s d r D$ & & & & & & & & & 20 & 0.88341 \\
\hline & SIRU21 & & & & & & & & & 13 & 0.85818 \\
\hline \multirow[t]{5}{*}{$2(153)$} & clfA & $c l f B$ & & & & & & & & 97 & 0.98954 \\
\hline & $s d r E$ & clfB & & & & & & & & 81 & 0.98577 \\
\hline & SIRU21 & $\operatorname{clfB}$ & & & & & & & & 79 & 0.98538 \\
\hline & SIRU05 & $\operatorname{clfB}$ & & & & & & & & 81 & 0.98491 \\
\hline & SIRU13 & $\operatorname{clfB}$ & & & & & & & & 80 & 0.98491 \\
\hline \multirow[t]{5}{*}{$3(816)$} & SIRU21 & clfA & $c l f B$ & & & & & & & 128 & 0.99599 \\
\hline & SAV1078 & clfA & $\operatorname{clfB}$ & & & & & & & 130 & 0.99583 \\
\hline & SIRU05 & clfA & $\operatorname{clfB}$ & & & & & & & 129 & 0.99576 \\
\hline & SAV1078 & SIRU21 & clfA & & & & & & & 125 & 0.99552 \\
\hline & $s d r D$ & clfB & clfA & & & & & & & 125 & 0.99544 \\
\hline \multirow[t]{5}{*}{$4(3060)$} & SIRU21 & SIRU05 & SIRU13 & $\operatorname{clfB}$ & & & & & & 143 & 0.99819 \\
\hline & SIRU21 & SAV1078 & clfA & $\operatorname{clfB}$ & & & & & & 144 & 0.99796 \\
\hline & SIRU21 & SAV1078 & clfA & $s d r D$ & & & & & & 141 & 0.99796 \\
\hline & SIRU21 & SIRU13 & clfA & $\operatorname{clfB}$ & & & & & & 141 & 0.99788 \\
\hline & SIRU21 & SAV1078 & $s d r E$ & $s d r D$ & & & & & & 140 & 0.99788 \\
\hline \multirow{5}{*}{$5(8568)$} & SIRU21 & SIRU05 & SIRU13 & $s d r D$ & $c l f B$ & & & & & 148 & 0.99889 \\
\hline & SIRU21 & SIRU05 & SIRU13 & clfA & $\operatorname{clfB}$ & & & & & 149 & 0.99874 \\
\hline & SIRU21 & SIRU05 & SIRU15 & SIRU13 & clfB & & & & & 148 & 0.99874 \\
\hline & SIRU21 & SAV1078 & clfA & $f n b B$ & $s d r D$ & & & & & 148 & 0.99874 \\
\hline & SIRU21 & SIRU05 & SIRU13 & $f n b A$ & clfB & & & & & 147 & 0.99866 \\
\hline \multirow[t]{5}{*}{$6(18564)$} & SIRU21 & SIRU05 & SIRU15 & SIRU13 & $\operatorname{clfB}$ & $s d r D$ & & & & 151 & 0.99921 \\
\hline & SIRU07 & SIRU21 & SIRU05 & SIRU13 & $\operatorname{clfB}$ & $s d r D$ & & & & 151 & 0.99914 \\
\hline & SIRU21 & SIRU05 & SIRU13 & clfA & clfB & $s d r D$ & & & & 151 & 0.99914 \\
\hline & SIRU21 & SIRU05 & SIRU13 & $f n b A$ & clfB & $s d r D$ & & & & 150 & 0.99914 \\
\hline & SIRU21 & SIRU13 & SAV1078 & $f n b B$ & $\operatorname{clf} A$ & $s d r D$ & & & & 150 & 0.99914 \\
\hline \multirow[t]{5}{*}{7 (31 824) } & SIRU07 & SIRU21 & SIRU05 & SIRU15 & SIRU13 & $\operatorname{clfB}$ & $s d r D$ & & & 153 & 0.99937 \\
\hline & SIRU21 & SIRU05 & SIRU13 & SAV1078 & $s d r D$ & $f n b B$ & clfA & & & 152 & 0.99937 \\
\hline & SIRU21 & SIRU05 & SIRU15 & SIRU13 & $f n b B$ & $c l f B$ & $s d r D$ & & & 152 & 0.99937 \\
\hline & SIRU21 & SIRU05 & SIRU15 & SIRU13 & $f n b A$ & $\operatorname{clfB}$ & $s d r D$ & & & 152 & 0.99937 \\
\hline & SIRU01 & SIRU21 & SIRU05 & SIRU15 & SIRU13 & clfB & $s d r D$ & & & 152 & 0.99929 \\
\hline \multirow[t]{5}{*}{$8(43758)$} & SIRU05 & SIRU07 & SIRU13 & SIRU15 & SIRU21 & $\operatorname{clfB}$ & $f n b B$ & $s d r D$ & & 154 & 0.99953 \\
\hline & SIRU05 & SIRU07 & SIRU13 & SIRU15 & SIRU21 & $\operatorname{clfB}$ & $f n b A$ & $s d r D$ & & 154 & 0.99953 \\
\hline & SIRU05 & SIRU01 & SIRU13 & SIRU15 & SIRU21 & clfB & $f n b B$ & $s d r D$ & & 153 & 0.99945 \\
\hline & SIRU05 & SIRU01 & SIRU13 & SIRU15 & SIRU21 & $\operatorname{clfB}$ & $f n b A$ & $s d r D$ & & 153 & 0.99945 \\
\hline & SIRU05 & SIRU07 & SIRU13 & SAV1078 & SIRU21 & clfA & $f n b B$ & $s d r D$ & & 153 & 0.99945 \\
\hline \multirow[t]{5}{*}{$9(48620)$} & SIRU01 & SIRU07 & SIRU21 & SIRU05 & SIRU15 & SIRU13 & $f n b B$ & $s d r D$ & $\operatorname{clfB}$ & 154 & 0.99953 \\
\hline & SIRU01 & SIRU07 & SIRU21 & SIRU05 & SIRU15 & SIRU13 & $f n b A$ & $s d r D$ & $\operatorname{clf} B$ & 154 & 0.99953 \\
\hline & SIRU07 & SIRU16 & SIRU21 & SIRU05 & SIRU15 & SIRU13 & $f n b B$ & $s d r D$ & $\operatorname{clf} B$ & 154 & 0.99953 \\
\hline & SIRU07 & SIRU16 & SIRU21 & SIRU05 & SIRU15 & SIRU13 & $f n b A$ & $s d r D$ & $\operatorname{clfB}$ & 154 & 0.99953 \\
\hline & SIRU07 & clfA & SIRU21 & SIRU05 & SIRU15 & SIRU13 & $f n b B$ & $s d r D$ & $\operatorname{clf} B$ & 154 & 0.99953 \\
\hline
\end{tabular}

${ }^{\star}$ Number of possible combinations for each set of loci are given in parentheses, i.e. there are 153 possible combinations if two out of the 18 loci are used.

(Table 4). Two MLVA profiles were observed among the seven patient isolates. They were the same as those from the food isolates, indicating that the $S$. aureus food poisoning outbreak in these seven patients was related to the consumption of the identified contaminated food. The isolates in this case investigation had sea, seb, sek and seq enterotoxin genes. These two examples demonstrated good concordance between the MLVA profiles of the S. aureus isolates from patients and those from infectious food sources. 


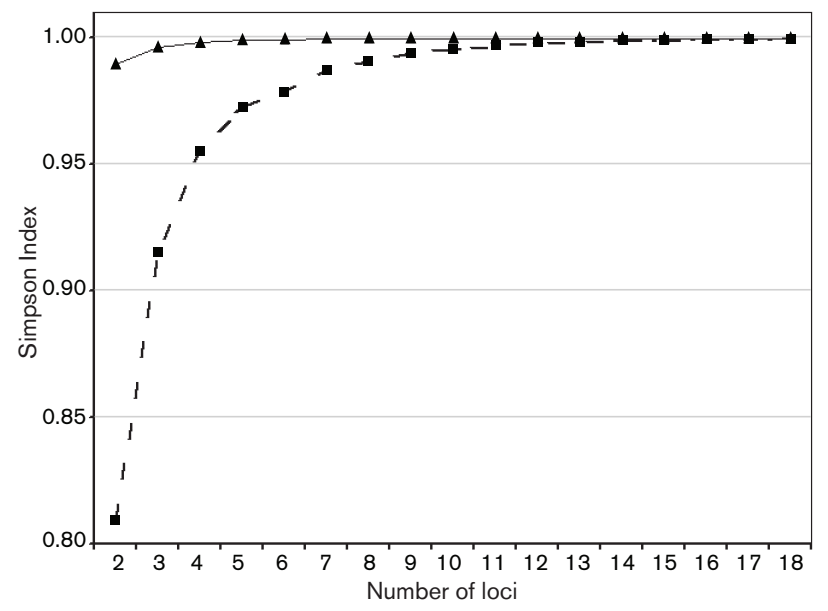

Fig. 2. Sl curves of the optimal loci $(\boldsymbol{\Lambda})$ and poorest loci combinations as determined by the OCF computer program.

\section{DISCUSSION}

In this study, the MLVA loci investigated were those most commonly used by various groups (Ikawaty et al., 2008; Melles et al., 2009; Pourcel et al., 2009; Sabat et al., 2003; Tenover et al., 2007). The precise size of amplicons was acquired using an ABI 3130 Genetic Analyzer, making loci comparison data more informative and portable. In addition, efforts were made to design primers to individually amplify variable loci with high sequence similarities, including $s d r C$, $s d r D$, and $s d r E$, and $f n b A$ and $f n b B$, whereas previously designed primers amplified all three $s d r C D E$ genes (Sabat et al., 2003) and both $f n b A B$ genes (Gilbert et al., 2006) in single reactions. The new primers for amplification of each individual gene have made it possible to use the variable property of these loci in the MLVA (Fig. 1).

The nomenclature for $S$. aureus alleles is confusing as authors express a null allele of a locus in different ways. For example, Hardy et al. (2004) used ' $\mathrm{x}$ ' and Ikawaty et al. (2008) used '999' to express a null allele, while Conceição et al. (2009) used '99' to express a null allele and '0' to express an amplicon that contained no tandem repeat unit but did contain the flanking sequences. A numerical nomenclature system was proposed to differentiate between no amplicon and amplicons containing no repeat units. The nomenclature system used in this study has made allele expression easier and more comprehensible and has enabled a computer program to analyse the data.

In the present study, the OFC computer program was developed to calculate the SI of a selected panel of loci, allowing investigators to gauge the efficacy of each combination. The OFC program was designed to calculate the minimal number of loci to get the maximal SI so that the optimal combination could be determined with a minimal work load. The maximum SI was achieved by using the optimal combination of eight loci (SIRU05, SIRU07, SIRU13, SIRU15, SIRU21, $c l f B, f n b B$ and $s d r D$ ) to get an SI of 0.999528 , which was as good as that obtained with the 18 loci combined. Significant savings in time and resources

Table 4. Profiles of the eight optimal loci for MLVA of isolates from the two outbreak case investigations

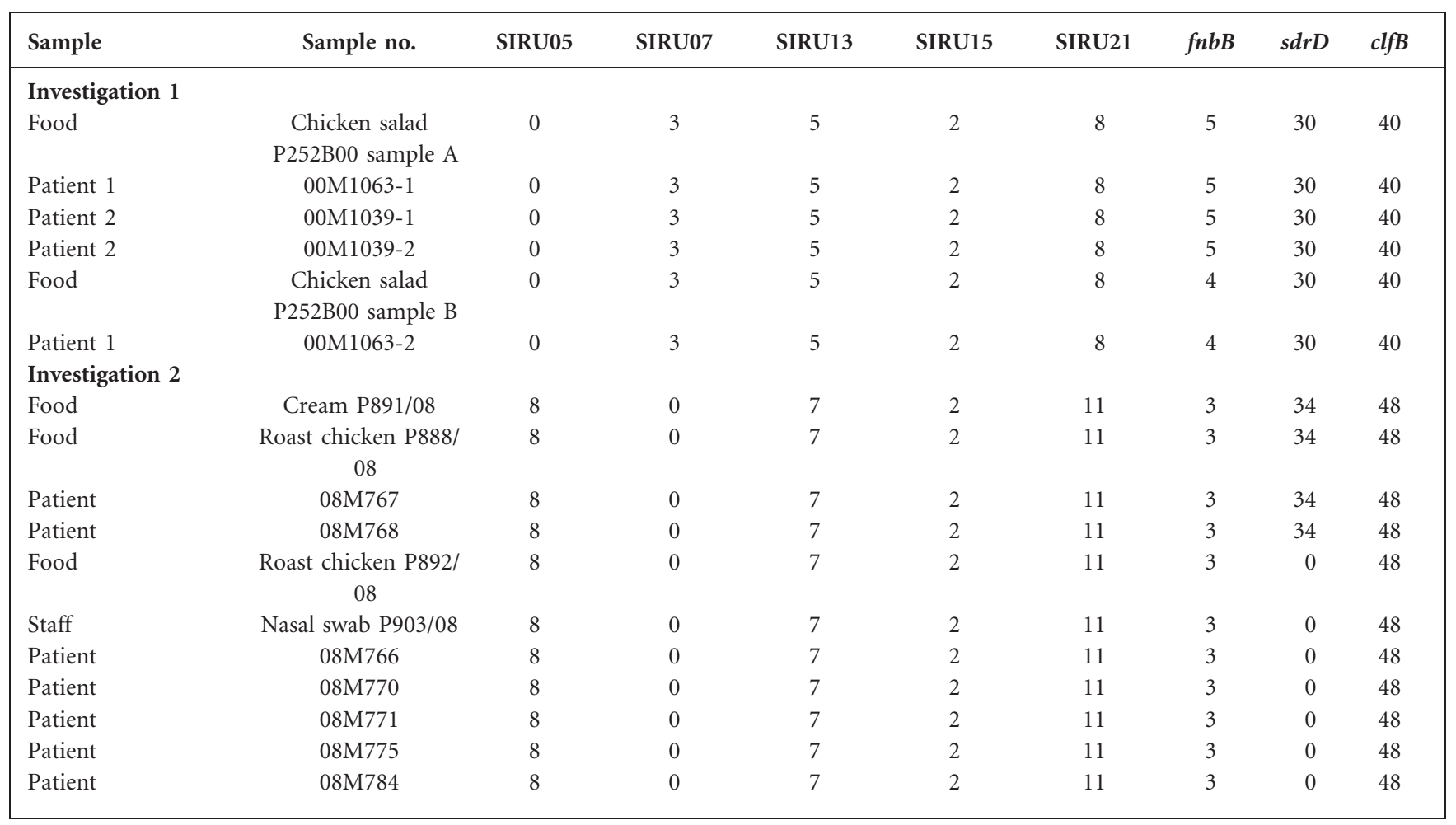


are possible using this optimized MLVA typing scheme. Another feature of this method is that it enables the epidemiologist to make a choice regarding the number of loci used for an outbreak investigation, depending on the level of discrimination required. For example, if a discriminatory level SI of 0.9959 was sufficient, only the optimal combination of the three loci SIRU21, clfA and clfB would be required (Table 3 ).

The OFC program can analyse both MLVA and binary data. It is not limited to $S$. aureus and could be useful for developing typing systems for other organisms. The program can also be readily implemented on personal computers. A maximum number of loci was set arbitrarily at 30 , considering the number of combinations created from one dataset. In addition, by setting an SI threshold, the program will ignore results that are less than the threshold, restricting the number of calculations that can be in excess of 65536 combinations. This figure is based on the maximum number of rows in Excel 2003. A plan has been made to upgrade the program to an internet accessible web server so that the calculation speeds can be significantly improved and users from wider academic and research communities can access and use this program.

Generally, there is a high level of variation in S. aureus populations. In the present study, 154 MLVA genotypes were observed out of 160 isolates. The level of diversity observed here was similar to that identified by PFGE and a SIRU-based MLVA assay, achieving 97 and 81 genotypes, respectively, out of 104 isolates tested (Conceição et al., 2009). Various combinations of MLVA loci chosen by researchers have produced SI $>0.95$ in previous reports. Schouls et al. (2009) used eight loci including sspA (VNTR09_01), SIRU21 (VNTR24_01), coa (VNTR81_01) and SAV1078 (VNTR63_01), giving an SI of 0.985. Ikawaty et al. (2008) used the six SIRU loci of Sabat et al. (2003) producing an SI of 0.987. Pourcel et al. (2009) developed two panels of loci for MLVA typing, the first panel consisting of 10 loci, which produced an SI of 0.9946 and the second panel consisting of an additional four loci that gave an SI of 0.9965. Interestingly, seven of these loci were the same as those in the present study, including SIRU21 $(=\mathrm{Sa} 0122), \quad$ coa $(=\mathrm{Sa} 0266), \quad 311 \quad(=\mathrm{Sa} 0311), \quad$ SIRU13 (=Sa1291), SIRU15 (=Sa1756), SAV1078 (=Sa1132) and SIRU01 (=Sa387). In the present study, the eight optimal loci that were identified produced the maximum diversity, with an SI of 0.9995. This result was similar to, or higher than, those of previously published data (Hardy et al., 2004; Pourcel et al., 2009; Schouls et al., 2009), the advantage being that the number of loci needed for typing has been reduced to a practical range, rather than 14 loci (Pourcel et al., 2009).

In an epidemiological outbreak investigation, samples with a one locus difference are conventionally regarded as related isolates using MLVA typing. The allele difference at one locus was thought to be a mutation occurring during the epidemic period (Torpdahl et al., 2007). In the two outbreak investigations tested here, there were mixed bacterial populations in the infectious food sources with different genotypes at $f n b B$ locus in the first investigation and at the $s d r D$ locus in the second investigation (Table 4). Interestingly, the isolates from infected patients also had two MLVA profiles, which corresponded with the profiles from the food sources. This suggests that, in outbreak investigations, the suspected food source may have contained mixed populations of $S$. aureus. There is a need to analyse a certain number of randomly selected colonies from suspected food sources to establish a connection between these isolates and mixed MLVA profiles from patient isolates. Therefore, most likely links between outbreak cases and suspected infectious food sources can be established. Although the optimized panel of eight loci worked well in linking outbreak patient isolates with the suspected food isolates in the two case studies, it is worth mentioning that there may be limitations in using this panel as some of the isolates used in this study to assess the 18 loci were from pet and zoo animals.

In conclusion, after screening the widely used 18 loci, eight of them (SIRUs 05, $-07,-13,-15$ and -21 and $c l f B, f n b B$ and $s d r D$ ) were identified by the OCF as the minimal number of loci required for optimal results. As such, the number of loci needed to produce the maximal differentiation of isolates has been reduced to a practical manageable range. A high-throughput $S$. aureus typing scheme like this one can provide rapid turnaround in outbreak investigations. The OFC computer program has helped us to identify the minimal number and optimal combination of loci for MLVA of $S$. aureus isolates and it may be of further use in developing typing schemes for other organisms.

\section{ACKNOWLEDGEMENTS}

The authors thank Ms Stephanie Ford of Idexx Laboratories, Coorparoo, QLD, Australia, for providing Staphylococcus aureus isolates from animals. The authors also thank the scientists and technicians in the Food Microbiology and Clinical Microbiology Sections of the Public Health Microbiology Laboratory, Queensland Health Forensic and Scientific Services, QLD, Australia, for isolating and storing the food-borne $S$. aureus isolates. X. Wang was supported by her employer, the Shijiazhuang Center for Disease Control and Prevention, Shijiazhuang, Hebei Province, China, for 1 year to be a visiting scientist at Queensland Health Forensic and Scientific Services, to whom we express our gratitude.

\section{REFERENCES}

Asao, T., Kumeda, Y., Kawai, T., Shibata, T., Oda, H., Haruki, K., Nakazawa, H. \& Kozaki, S. (2003). An extensive outbreak of staphylococcal food poisoning due to low-fat milk in Japan: estimation of enterotoxin $\mathrm{A}$ in the incriminated milk and powdered skim milk. Epidemiol Infect 130, 33-40.

Bannerman, T. \& Peacock, S. (2007). Staphylococcus, Micrococcus and other catalase-positive cocci. In Manual of Clinical Microbiology, 9th edn, vol. 1, pp. 390-411. Edited by P. R. Murray, E. J. Baron, J. H. Jorgensen, M. L. Landry \& M. A. Pfaller. Washington, DC: American Society for Microbiology. 
Conceição, T., Aires de Sousa, M. \& de Lencastre, H. (2009). Staphylococcal interspersed repeat unit typing of Staphylococcus aureus: evaluation of a new multilocus variable-number tandemrepeat analysis typing method. J Clin Microbiol 47, 1300-1308.

Coombs, G. W., Nimmo, G. R., Pearson, J. C., Christiansen, K. J., Bell, J. M., Collignon, P. J., McLaws, M. L. \& Australian Group for Antimicrobial Resistance (2009). Prevalence of MRSA strains among Staphylococcus aureus isolated from outpatients, 2006. Commun Dis Intell 33, 10-20.

Cowell, N. A., Hansen, M. T., Langley, A. J., Graham, T. M. \& Bates, J. R. (2002). Outbreak of staphylococcal enterotoxin food poisoning. Commun Dis Intell 26, 574-575.

Francois, P., Huyghe, A., Charbonnier, Y., Bento, M., Herzig, S., Topolski, I., Fleury, B., Lew, D., Vaudaux, P. \& other authors (2005). Use of an automated multiple-locus, variable-number tandem repeat-based method for rapid and high-throughput genotyping of Staphylococcus aureus isolates. J Clin Microbiol 43, 33463355.

Fullerton, K. \& OzFoodNet Working Group (2009). OzFoodNet quarterly report, 1 October to 31 December 2008. Commun Dis Intell 33, 53-58.

Gilbert, F. B., Fromageau, A., Gélineau, L. \& Poutrel, B. (2006). Differentiation of bovine Staphylococcus aureus isolates by use of polymorphic tandem repeat typing. Vet Microbiol 117, 297-303.

Grissa, I., Bouchon, P., Pourcel, C. \& Vergnaud, G. (2008). On-line resources for bacterial micro-evolution studies using MLVA or CRISPR typing. Biochimie 90, 660-668.

Hardy, K. J., Ussery, D. W., Oppenheim, B. A. \& Hawkey, P. M. (2004). Distribution and characterization of staphylococcal interspersed repeat units (SIRUs) and potential use for strain differentiation. Microbiology 150, 4045-4052.

Hardy, K. J., Oppenheim, B. A., Gossain, S., Gao, F. \& Hawkey, P. M. (2006). Use of variations in staphylococcal interspersed repeat units for molecular typing of methicillin-resistant Staphylococcus aureus strains. J Clin Microbiol 44, 271-273.

Hunter, P. R. \& Gaston, M. A. (1988). Numerical index of the discriminatory ability of typing systems: an application of Simpson's index of diversity. J Clin Microbiol 26, 2465-2466.

Hwang, S. Y., Kim, S. H., Jang, E. J., Kwon, N. H., Park, Y. K., Koo, H. C., Jung, W. K., Kim, J. M. \& Park, Y. H. (2007). Novel multiplex PCR for the detection of the Staphylococcus aureus superantigen and its application to raw meat isolates in Korea. Int J Food Microbiol 117, 99-105.

Ikawaty, R., Willems, R. J., Box, A. T., Verhoef, J. \& Fluit, A. C. (2008). Novel multiple-locus variable-number tandem-repeat analysis method for rapid molecular typing of human Staphylococcus aureus. J Clin Microbiol 46, 3147-3151.

Le Loir, Y., Baron, F. \& Gautier, M. (2003). Staphylococcus aureus and food poisoning. Genet Mol Res 2, 63-76.
Lindstedt, B. A. (2005). Multiple-locus variable number tandem repeats analysis for genetic fingerprinting of pathogenic bacteria. Electrophoresis 26, 2567-2582.

Malachowa, N., Sabat, A., Gniadkowski, M., Krzyszton-Russjan, J., Empel, J., Miedzobrodzki, J., Kosowska-Shick, K., Appelbaum, P. C. \& Hryniewicz, W. (2005). Comparison of multiple-locus variablenumber tandem-repeat analysis with pulsed-field gel electrophoresis, spa typing, and multilocus sequence typing for clonal characterization of Staphylococcus aureus isolates. J Clin Microbiol 43, 3095-3100.

Melles, D. C., Schouls, L., François, P., Herzig, S., Verbrugh, H. A., van Belkum, A. \& Schrenzel, J. (2009). High-throughput typing of Staphylococcus aureus by amplified fragment length polymorphism (AFLP) or multi-locus variable number of tandem repeat analysis (MLVA) reveals consistent strain relatedness. Eur J Clin Microbiol Infect Dis 28, 39-45.

Pourcel, C., Hormigos, K., Onteniente, L., Sakwinska, O., Deurenberg, R. H. \& Vergnaud, G. (2009). Improved multiple-locus variable-number tandem-repeat assay for Staphylococcus aureus genotyping, providing a highly informative technique together with strong phylogenetic value. J Clin Microbiol 47, 3121-3128.

Sabat, A., Krzyszton-Russjan, J., Strzalka, W., Filipek, R., Kosowska, K., Hryniewicz, W., Travis, J. \& Potempa, J. (2003). New method for typing Staphylococcus aureus strains: multiple-locus variable-number tandem repeat analysis of polymorphism and genetic relationships of clinical isolates. J Clin Microbiol 41, 1801-1804.

Schmitt, M., Schuler-Schmid, U. \& Schmidt-Lorenz, W. (1990). Temperature limits of growth, TNase and enterotoxin production of Staphylococcus aureus strains isolated from foods. Int J Food Microbiol 11, 1-19.

Schouls, L. M., Spalburg, E. C., van Luit, M., Huijsdens, X. W., Pluister, G. N., van Santen-Verheuvel, M. G., van der Heide, H. G., Grundmann, H., Heck, M. E. \& de Neeling, A. J. (2009). Multiplelocus variable number tandem repeat analysis of Staphylococcus aureus: comparison with pulsed-field gel electrophoresis and spatyping. PLoS ONE 4, e5082.

Tenover, F. C., Vaughn, R. R., McDougal, L. K., Fosheim, G. E. \& McGowan, J. E., Jr (2007). Multiple-locus variable-number tandemrepeat assay analysis of methicillin-resistant Staphylococcus aureus strains. J Clin Microbiol 45, 2215-2219.

Torpdahl, M., Sørensen, G., Lindstedt, B. A. \& Nielsen, E. M. (2007). Tandem repeat analysis for surveillance of human Salmonella Typhimurium infections. Emerg Infect Dis 13, 388-395.

van Belkum, A. (2007). Tracing isolates of bacterial species by multilocus variable number of tandem repeat analysis (MLVA). FEMS Immunol Med Microbiol 49, 22-27.

Vindel, A., Cuevas, O., Cercenado, E., Marcos, C., Bautista, V., Castellares, C., Trincado, P., Boquete, T., Pérez-Vázquez, M. \& other authors (2009). Methicillin-resistant Staphylococcus aureus in Spain: molecular epidemiology and utility of different typing methods. J Clin Microbiol 47, 1620-1627. 